\title{
Fundamentals of Anesthesia Care: Competency-Based Learning Modules for the Health Professions
}

\author{
Judith A. Littleford (ed). Art Bookbindery, 2009, 393 pp, ISBN 978-0-9811045-0-8
}

\author{
Anne Wong, MD
}

Published online: 25 June 2009

(C) Canadian Anesthesiologists' Society 2009

This book was originally developed as the curriculum for the University of Manitoba's Anesthesia Clinical Assistant Program. Over 100 authors have contributed to the book's 165 teaching modules, which are organized into eleven broad sections: Airway, Anesthesia Considerations, Assessment, Equipment, Cannulation and Injection Procedures, Monitors, Pharmacology and Physiology, Clinical Management, Pediatric Anesthesia, Regional Anesthesia and Pain Management Protocols, and Policies and Guidelines. In general, each teaching module is similarly organized. Each module begins with learning objectives that outline core knowledge. The objectives are followed by several pertinent references (from textbooks, journal articles, or websites), and after the reference section is a list of competencies representing the requisite clinical skills. Each module also includes a few multiple choice questions as well as a set of advanced topics for consideration.

This book provides comprehensive coverage of the topics in anesthesia practice. However, there are some inconsistencies in the effectiveness of the various teaching modules. For instance, the degree of detail in the learning objectives and competencies varies from descriptions that are succinct to those that are over a page in length. Some material tends to be basic and redundant (e.g., a full page description of Korotkoff sounds, p. 216), while material in other sections is esoteric and beyond the scope of practice of an anesthesia assistant (e.g., consideration of succinylcholine for stridor, p. 315). As well, some of the authors combine teaching material with their objectives, which can distract the reader. Although the modules are designed to be independent, the order of topics can be counter-intuitive, for example, in the Airway section, advanced airway modules, such as awake fibreoptic intubation, are presented before the modules pertaining to preoxygenation and basic orotracheal intubation. Unfortunately, the book does not address the evaluation of competencies.

Despite the aforementioned limitations, Fundamentals of Anesthesia Care: Competency-Based Learning Modules for the Health Professions represents a unique contribution to addressing anesthesia assistant training in Canada. Although some modules provide teaching material, this book cannot be used as a stand-alone text, as most of the teaching information is referenced to outside sources. As a teaching manual, its size and detail may make it unwieldy for general use, and teachers will have to be selective in their use of the learning material. The book's major strength lies in its broad coverage of the scope of anesthetic practice and its delineation of the clinical competencies of an anesthesia assistant. Also, this book may be useful for advanced learners from other disciplines and beneficial as a reference guide to aid teachers in curriculum development.

\footnotetext{
A. Wong, MD ( $\square)$

St. Joseph's Healthcare, McMaster University,

Hamilton, ON, Canada

e-mail: wongan@mcmaster.ca
} 